\title{
Cefamandole Nafate
}

National Cancer Institute

\section{Source}

National Cancer Institute. Cefamandole Nafate. NCI Thesaurus. Code C47966.

The sodium salt form of cefamandole formyl ester. Cefamandole nafate is a pro-drug that is hydrolyzed by plasma esterases to produce cefamandole, a semi-synthetic betalactam, second-generation cephalosporin antibiotic with bactericidal activity.

Cefamandole binds to and inactivates penicillin-binding proteins (PBP) located on the inner membrane of the bacterial cell wall. Inactivation of PBPs interferes with the crosslinkage of peptidog lycan chains necessary for bacterial cell wall strength and rigidity. This results in the weakening of the bacterial cell wall and causes cell lysis. 